\title{
Limitations of global end-diastolic volume index as a parameter of cardiac preload in the early phase of severe sepsis: a subgroup analysis of a multicenter, prospective observational study
}

Tomoyuki Endo ${ }^{1 *}$, Shigeki Kushimoto ${ }^{2}$, Satoshi Yamanouchi' ${ }^{2}$, Teruo Sakamoto ${ }^{3}$, Hiroyasu Ishikura ${ }^{4}$, Yasuhide Kitazawa ${ }^{5}$, Yasuhiko Taira ${ }^{6}$, Kazuo Okuchi $^{7}$, Takashi Tagami ${ }^{8}$, Akihiro Watanabe $^{8}$, Junko Yamaguchi ${ }^{9}$, Kazuhide Yoshikawa ${ }^{10}$, Manabu Sugita ${ }^{11}$, Yoichi Kase ${ }^{12}$, Takashi Kanemura ${ }^{13}$, Hiroyuki Takahashi ${ }^{14}$, Yuuichi Kuroki ${ }^{15}$, Hiroo Izumino ${ }^{16}$, Hiroshi Rinka ${ }^{17}$, Ryutarou Seo ${ }^{18}$, Makoto Takatori ${ }^{19}$, Tadashi Kaneko ${ }^{20}$, Toshiaki Nakamura ${ }^{21}$, Takayuki Irahara ${ }^{22}$, Nobuyuki Saito ${ }^{23}$ and PiCCO Pulmonary Edema Study Group

\begin{abstract}
Background: In patients with severe sepsis, depression of cardiac performance is common and is often associated with left ventricular (LV) dilatation to maintain stroke volume. Although it is essential to optimize cardiac preload to maintain tissue perfusion in patients with severe sepsis, the optimal preload remains unknown. This study aimed to evaluate the reliability of global end-diastolic volume index (GEDI) as a parameter of cardiac preload in the early phase of severe sepsis.
\end{abstract}

Methods: Ninety-three mechanically ventilated patients with acute lung injury/acute respiratory distress syndrome secondary to sepsis were enrolled for subgroup analysis in a multicenter, prospective, observational study. Patients were divided into two groups - with sepsis-induced myocardial dysfunction (SIMD) and without SIMD (non-SIMD) according to a threshold LV ejection fraction (LVEF) of 50\% on the day of enrollment. Both groups were further subdivided according to a threshold stroke volume variation (SW) of $13 \%$ as a parameter of fluid responsiveness.

Results: On the day of enrollment, there was a positive correlation ( $r=0.421, p=0.045)$ between GEDI and SW in the SIMD group, whereas this paradoxical correlation was not found in the non-SIMD group and both groups on day 2. To evaluate the relationship between attainment of cardiac preload optimization and GEDI value, GEDI with SW $\leq 13 \%$ and SW $>13 \%$ was compared in both the SIMD and non-SIMD groups. SW $\leq 13 \%$ implies the attainment of cardiac preload optimization. Among patients with SIMD, GEDI was higher in patients with SW $>13 \%$ than in patients with SW $\leq 13 \%$ on the day of enrollment (872 [785-996] $\mathrm{mL} / \mathrm{m}^{2}$ vs. 640 [597-696] $\left.\mathrm{mL} / \mathrm{m}^{2} ; p<0.001\right)$; this finding differed from the generally recognized relationship between GEDI and SW. However, GEDI was not significantly different between patients with SW $\leq 13 \%$ and SW $>13 \%$ in the non-SIMD group on the day of enrollment and both groups on day 2.

Conclusions: In the early phase of severe sepsis in mechanically ventilated patients, there was no constant relationship between GEDI and fluid reserve responsiveness, irrespective of the presence of SIMD. GEDI should be used as a cardiac preload parameter with awareness of its limitations.

Keywords: Sepsis-induced myocardial dysfunction, Global end-diastolic volume index, Stroke volume variation, Diastolic dysfunction, Severe sepsis

\footnotetext{
* Correspondence: entomo@med.tohoku.ac.jp

${ }^{1}$ Department of Emergency and Critical Care Medicine, Tohoku University

Hospital, Sendai, Miyagi 980-8574, Japan

Full list of author information is available at the end of the article
} 


\section{Background}

In patients with severe sepsis, depression of cardiac performance has been described for more than three decades. Such sepsis-induced myocardial dysfunction (SIMD) is acute, is reversible, and has a high incidence of approximately $40 \%$ in patients with severe sepsis [1-5].

Although the relationship between SIMD and left ventricular (LV) dilatation has been demonstrated, the effect of compensatory LV dilatation is controversial in the clinical setting. Several studies have reported that LV dilatation, which means increased LV compliance, might be a protective mechanism associated with better survival in patients with reduced LV ejection fraction (LVEF) [1,5-7]. In contrast, a study indicated that such LV dysfunction was related to poor prognosis [8]. In addition to LV dilatation, the right ventricle has also been shown to be dilated because of right ventricular dysfunction in SIMD $[1,3,4,9,10]$. Therefore, global enddiastolic volume would be increased by biventricular dysfunction in SIMD.

Although it is essential to optimize cardiac preload immediately to maintain tissue perfusion in patients with severe sepsis and signs of tissue hypoperfusion, it is difficult to appropriately evaluate the status of cardiac preload in patients with SIMD during the early phase of severe sepsis [11]; moreover, a reliable parameter for cardiac preload has not been elucidated thus far. Recently, the transpulmonary thermodilution (TD) system has been widely used in the critical care setting. Global enddiastolic volume index (GEDI), a static volumetric parameter in TD, is considered more eligible for determining cardiac preload than central venous pressure or pulmonary artery wedge pressure in patients with septic shock [12]. Despite the usefulness of GEDI as a preload parameter, the optimal value of GEDI in patients with SIMD has not been investigated and is expected to be increased compared to that in sepsis patients with preserved cardiac function. We hypothesized that the optimal GEDI, as the preload parameter, would be greater in patients with SIMD than in those without SIMD. In the present study, we aimed to evaluate the difference between GEDI in patients with and without SIMD and to determine the reliability of GEDI as a parameter of cardiac preload during the early phase of severe sepsis.

\section{Methods}

This is a subgroup analysis of a multicenter, prospective, observational study conducted to clarify the clinical features of acute respiratory distress syndrome (ARDS) and to establish quantitative diagnostic criteria [13]. The detailed study protocol has been described in the original article. The study was approved by the ethics committee of each of the 23 institutions, and written informed consent was provided by each patient's next of kin. The investigation was registered with the University Hospital Medical Information Network (UMIN) Clinical Trials Registry, UMIN-CTR ID UMIN000003627. Between March 2009 and August 2011, 301 patients were enrolled in this study. The inclusion criteria were age $\geq 15$ years, requirement of mechanical ventilation (expected $>48 \mathrm{~h}$ ) for acute respiratory failure with a partial pressure of arterial oxygen $\left(\mathrm{PaO}_{2}\right) /$ fraction of inspired oxygen $\left(\mathrm{FiO}_{2}\right)$ ratio of $\leq 300 \mathrm{mmHg}$, and bilateral infiltration on chest radiography. Patients with a history of pulmonary resection, pulmonary thromboembolism, severe peripheral arterial disease, a cardiac index of $<1.5 \mathrm{~L} / \mathrm{min} / \mathrm{m}^{2}$, lung contusion, burns, and other conditions unsuitable for evaluation with the TD technique were excluded. Data sampling was performed once a day for 3 days. Among the 301 patients, 207 were considered to have acute lung injury (ALI)/ ARDS. Ninety-three patients with ALI/ARDS secondary to sepsis whose LVEF was evaluated by transthoracic echocardiography on the day of enrollment were enrolled for this subgroup analysis. Because the method to measure LVEF was not predetermined, each institution had selected either the single-plane area-length method or modified Simpson method according to the presence of LV asynergy. Depending on the human resources of each institution, a registered medical sonographer, a cardiologist, an intensivist, or an emergency physician performed the LVEF measurement. Patients were divided into two groups according to LVEF on the day of enrollment. In this study, the threshold value of LVEF with SIMD was defined as $50 \%$, as previously described $[3,8,14]$, which was sufficiently lower than the normal echocardiographic LVEF of $66 \% \pm 5 \%(\mathrm{SD})$ in the Japanese population [15]. To investigate the fluid reserve responsiveness in these patients, both groups were further subdivided into two subgroups according to stroke volume variation (SVV), and SVV threshold was defined as $13 \%$, as previously described $[16,17]$. In mechanically ventilated patients, SVV is a useful predictor of fluid responsiveness and has better sensitivity and specificity than GEDI [18-24]. SVV $\leq 13 \%$ indicates low fluid reserve responsiveness and the attainment of cardiac preload optimization.

\section{Measurement of GEDI, EVLWi, PVPI, and SVV}

A thermistor-tipped catheter was connected to the $\mathrm{PiCCO}^{\circ}$ plus or $\mathrm{PiCCO}^{\circ} 2$ monitor (Pulsion Medical Systems, Munich, Germany). This monitor uses a singlethermal indicator technique to calculate the cardiac output, global end-diastolic volume (GEDV), and extravascular lung water (EVLW). GEDV is calculated as the difference between the intrathoracic thermal volume and pulmonary thermal volume, which represents the combined end-diastolic volumes of the four cardiac chambers. Intrathoracic blood volume was calculated as $1.25 \times$ GEDV - 28.4 [25]. EVLW is the difference between the 
intrathoracic thermal volume and intrathoracic blood volume [25]. Pulmonary vascular permeability index (PVPI) was calculated as the ratio of EVLW to pulmonary blood volume [26]. The absolute EVLW and GEDV values were indexed to predicted body weight [27-29] and provided as extravascular lung water index (EVLWi) and GEDI, respectively. The $\mathrm{PiCCO}^{\circ}$ plus or $\mathrm{PiCCO}^{\circ} 2$ monitor can also automatically calculate the SVV, which is a dynamic parameter of fluid responsiveness [18-24]. We recorded the SVV value at the time of GEDI measurement.

\section{Statistical analysis}

Data are presented as median (interquartile range (IQR)). The proportions were compared using Pearson's chisquare test or Fisher's exact test, as required. Spearman's rank correlation coefficient was used for determining the correlation between variables, and Mann-Whitney's $U$ test was used for assessing the differences between groups. A $p$ value $<0.05$ was considered significant. All statistical analyses were performed using SPSS 19.0 for Windows (SPSS, Chicago, IL, USA).

\section{Results}

\section{Characteristics of patients with ALI/ARDS secondary to} sepsis on the day of enrollment

Table 1 shows the patient characteristics on the day of enrollment. Ninety-three patients were evaluated for inclusion in the current analysis. Of those, 23 patients were diagnosed with SIMD, which was defined as $\mathrm{LVEF} \leq 50 \%$.

Table 1 Characteristics of patients with ALI/ARDS secondary to sepsis on the day of enrollment

\begin{tabular}{lccc}
\hline Characteristics & SIMD group & $\begin{array}{c}\text { Non-SIMD } \\
\text { group } \\
\mathbf{n = 7 0}\end{array}$ & $\boldsymbol{p}$ value \\
\hline Age, years & $79(64-83)$ & $72(62-82)$ & 0.430 \\
Gender (male), $n$ (\%) & $15(65.2)$ & $42(60.0)$ & 0.806 \\
Septic shock, $n$ (\%) & $14(60.9)$ & $40(57.1)$ & 0.899 \\
Vasoactive-inotropic agents, & $21(91.3)$ & $50(71.4)$ & 0.087 \\
$n$ (\%) & $24(19-27)$ & $25(19-30)$ & 0.565 \\
APACHE II score, points & $12(10-14)$ & $11(9-13)$ & 0.345 \\
SOFA score, points & $128(80-198)$ & $134(96-185)$ & 0.943 \\
PaO $/$ /FIO ${ }_{2}$ ratio, mmHg & $757(644-892)$ & $837(669-934)$ & 0.293 \\
GEDI, mL/m ${ }^{2}$ & $16.2(13.2-21.5)$ & $18.2(14.2-23.6)$ & 0.287 \\
EVLWi, mL/kg & $2.9(2.4-3.7)$ & $3.1(2.4-3.9)$ & 0.599 \\
PVPI & $9(39.1)$ & $32(45.7)$ & 0.635 \\
28-day mortality, $n$ (\%) & &
\end{tabular}

All data are presented as median (interquartile range) unless otherwise stated. ALI/ARDS acute lung injury/acute respiratory distress syndrome, APACHE Acute Physiology and Chronic Health Evaluation, SIMD sepsis-induced myocardial dysfunction, SOFA Sequential Organ Failure Assessment, GEDI global end-diastolic volume index, EVLWi extravascular lung water index, PVPI pulmonary vascular permeability index.
There were no significant differences in age, gender, prevalence of septic shock, APACHE II score, SOFA score, $\mathrm{PaO}_{2} / \mathrm{FiO}_{2}$ ratio, GEDI, EVLWi, PVPI, and 28-day mortality between patients with SIMD and without SIMD (non-SIMD).

\section{Relationship between GEDI, SVV, and LVEF}

Initially, we analyzed the relationship between GEDI and SVV in both the SIMD and non-SIMD groups (Figures 1 and 2). On the day of enrollment (Figure 1), there was a moderate positive correlation $(r=0.421, \quad p=0.045)$ between GEDI and SVV in the SIMD group, whereas this correlation was not found in the non-SIMD group $(r=-0.081, p=0.503)$. On day 2 (Figure 2), there were no correlations between GEDI and SVV in both groups (SIMD, $r=-0.148, p=0.557$; non-SIMD, $r=-0.083, p=0.545$ ).

To evaluate the relationship between attainment of cardiac preload optimization and GEDI value, we compared GEDI between patients with SVV $\leq 13 \%$ and SVV $>13 \%$ in both the SIMD and non-SIMD groups (Figures 3 and 4). Among the patients with SIMD, GEDI was significantly higher in those with SVV $>13 \%$ than in those with SVV $\leq 13 \%$ on the day of enrollment (SVV $>13 \%$, 872 [785-996] mL/m²; SVV $\leq 13 \%, 640$ [597-696] mL/m²; $p<0.001)$. In contrast, GEDI was not significantly different between patients with SVV $\leq 13 \%$ and SVV $>13 \%$ in the non-SIMD group on the day of enrollment (SVV $\leq 13 \%, 852$ [687-934] $\mathrm{mL} / \mathrm{m}^{2} ; \mathrm{SVV}>13 \%, 787$ [620-998] $\mathrm{mL} / \mathrm{m}^{2} ; p=0.629$ ) (Figure 3). On day 2, in both the SIMD and non-SIMD groups, there were no significant differences in the levels of GEDI between patients with SVV $\leq 13 \%$ and SVV $>13 \%$ (SIMD with SVV $\leq 13 \%$, 881 [784-1001] $\mathrm{mL} / \mathrm{m}^{2}$ vs. SIMD with $\mathrm{SVV}>13 \%, 811$ [724-1062] $\mathrm{mL} / \mathrm{m}^{2}, p=0.722$; non-SIMD with SVV $\leq 13 \%$, 853 [754-1170] $\mathrm{mL} / \mathrm{m}^{2}$ vs. non-SIMD with $\mathrm{SVV}>13 \%$, 795 [730-1007] $\mathrm{mL} / \mathrm{m}^{2}, p=0.289$ ) (Figure 4).

\section{Discussion}

Parker et al. published a landmark article in the early 1980s demonstrating SIMD [5]. They suggested that adequate LV stroke output can be maintained through acute compensatory LV dilatation despite a severely depressed LVEF, which was calculated using radionuclide cineangiography. The methods used for evaluating cardiac preload and LVEF in sepsis patients have changed from cineangiography to pulmonary artery catheterization, echocardiography, and TD; these methods can be used to obtain accurate LV volume measurements at the bedside [30]. Although Parker et al. demonstrated 100\% LV dilatation (from 100 to $200 \mathrm{~mL}$ ) in patients with systolic dysfunction using cineangiography [5], other investigators have reported that the rate of increase in LV end-diastolic volume is less than $20 \%$ using echocardiography $[3,6]$. These discordant findings may be because both the right and LV 

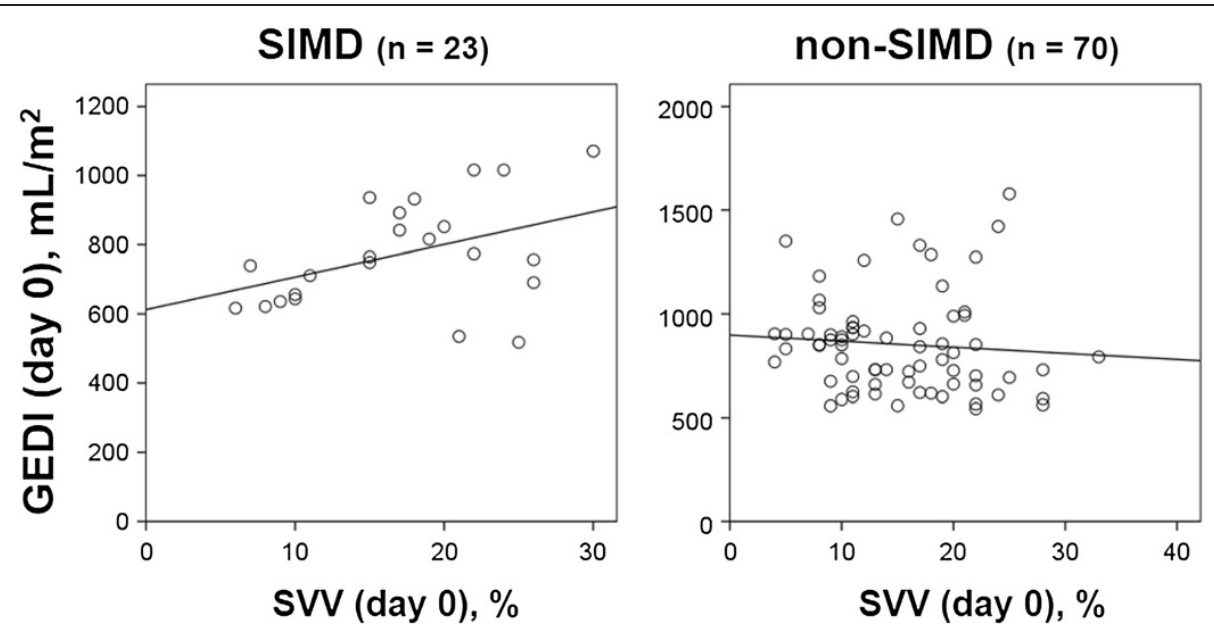

Figure 1 Relationship between SVV and GEDI on the day of enrollment (day 0). There was a moderate positive correlation $(r=0.421$, $p=0.045)$ between GEDI and SW in the SIMD group, whereas no such correlation was noted in the non-SIMD group $(r=-0.081, p=0.503)$. SIMD, sepsis-induced myocardial dysfunction; GEDI, global end-diastolic volume index; SW, stroke volume variation.

chambers are enclosed in the relatively stiff pericardium that restricts expansion of the entire heart. For such compensatory dilated heart, the fluid reserve responsiveness and the optimal GEDI value that does not cause left atrial hypertension are still unknown. Dynamic hemodynamic parameters such as SVV and pulse pressure variation (PPV) are now available; these parameters indicate the part of the Frank-Starling curve in which the cardiac preload is located [24]. If SVV/PPV shows $\leq 13 \%$ variation, there is low fluid reserve responsiveness, and the cardiac preload may be located on the plateau part of the FrankStarling curve $[16,17]$. Therefore, by evaluating GEDI and SVV simultaneously, we could estimate whether the measured GEDI was located on the plateau part of the Frank-
Starling curve. Considering the abovementioned findings, in this study, we analyzed the relationship between LVEF, GEDI, and SVV in order to evaluate the reliability of GEDI as a parameter of cardiac preload in the early phase of severe sepsis.

In an intensive care unit setting, it is necessary to consider extracardiac factors such as positive airway pressure ventilation, pulmonary hypertension secondary to lung injury, and afterload alteration by vasopressors; hence, the proposed GEDI reference range may not be suitable for such critically ill patients. Recently, Eichhorn et al. analyzed the published data for GEDI between a cohort of septic patients and patients undergoing major surgery [31]. In their analysis, the pooled estimate for
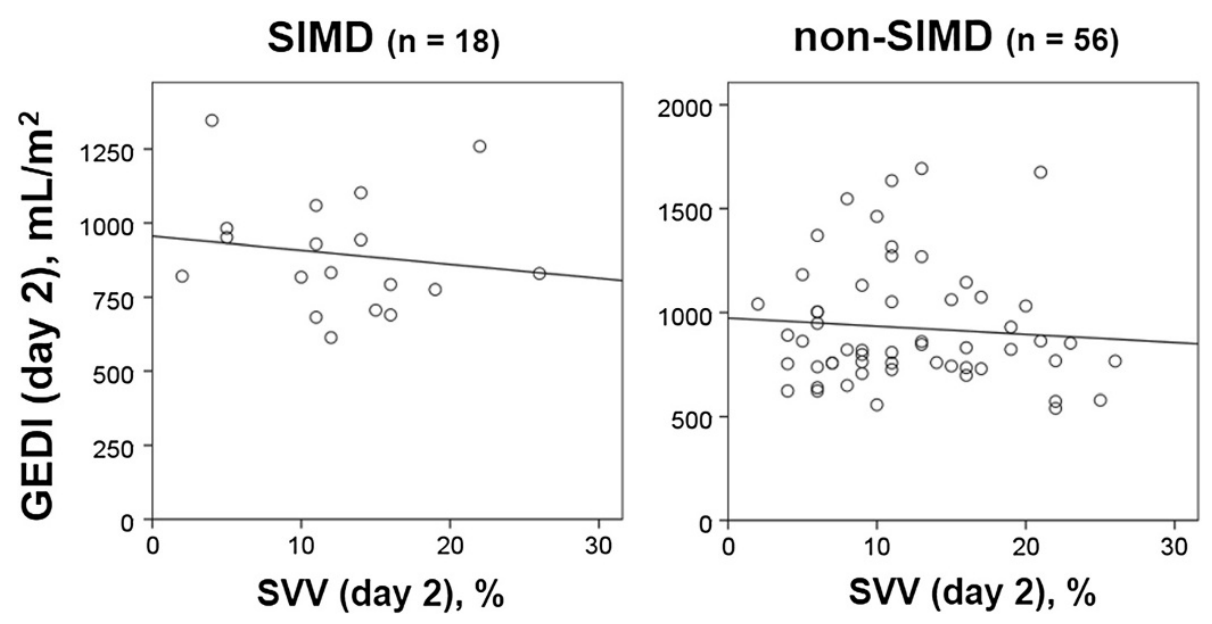

Figure 2 Relationship between SVV and GEDI on day 2. There were no correlations between GEDI and SW in both groups (SIMD group: $r=-0.148, p=0.557$; non-SIMD group: $r=-0.083, p=0.545$ ). SIMD, sepsis-induced myocardial dysfunction; GEDI, global end-diastolic volume index; SW, stroke volume variation. 


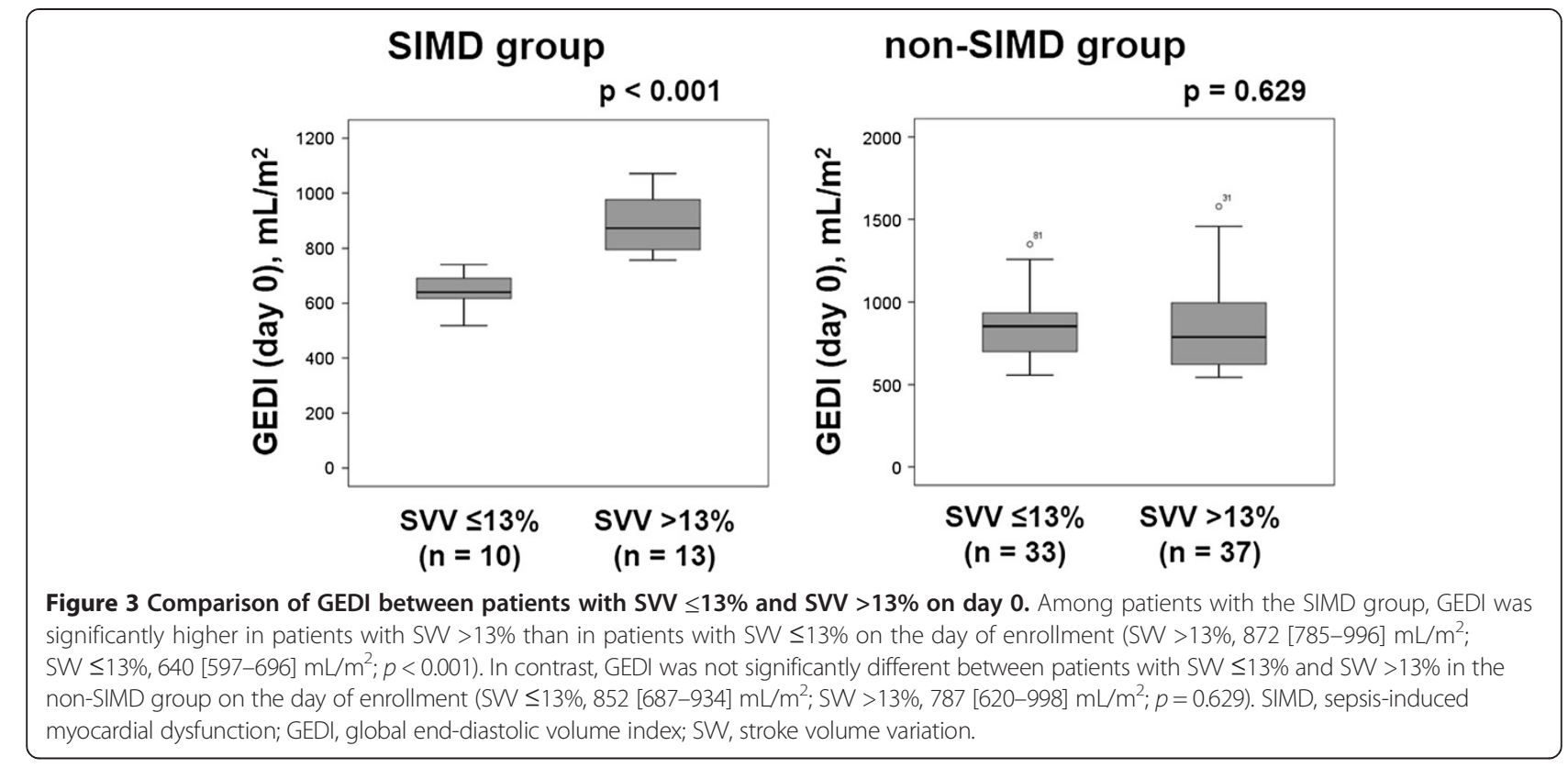

the mean value for GEDI was $788 \mathrm{~mL} / \mathrm{m}^{2}$ in septic patients and $694 \mathrm{~mL} / \mathrm{m}^{2}$ in surgical patients; this finding suggested that the mean GEDI was significantly higher in sepsis patients than in the surgical group. Despite this finding, the optimal GEDI value in patients with sepsis and those with SIMD is still unknown.

At present, several methods are available to evaluate myocardial dysfunction, such as cineangiography, cinescintigraphy, magnetic resonance imaging, cardiac index measured by TD, and echocardiography. Among those methods, both cardiac index by TD and echocardiography are useful for the bedside evaluation of mechanically ventilated patients. Although cardiac index can be measured continuously by the TD system as a hemodynamic parameter, it could be affected by alteration of heart rate, preload, afterload, right ventricular function, and LV compliance. Moreover, cardiac index would not necessarily be depressed in sepsis with SIMD $[1,3,8]$. We considered that echocardiography would be more eligible for this study as it had been widely used in previous studies to evaluate the presence of SIMD and could directly visualize the cardiac performance $[1,3,4,6-8,10,14]$. Therefore, we
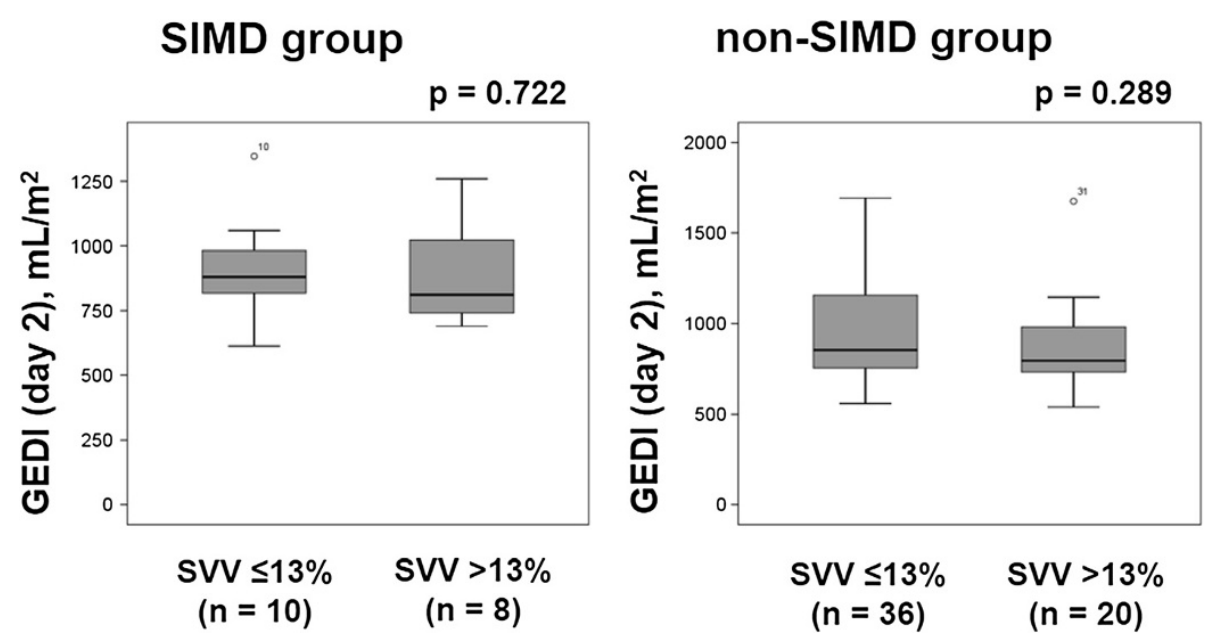

Figure 4 Comparison of GEDI between patients with SVV $\leq 13 \%$ and SVV $>13 \%$ on day 2 . On day 2 , in both the SIMD and non-SIMD groups, there were no significant differences in the levels of GEDI between patients with SW $\leq 13 \%$ and SW $>13 \%$ (SIMD with SW $\leq 13 \%, 881$ $[784-1,001] \mathrm{mL} / \mathrm{m}^{2}$ vs. SIMD with SW >13\%, $811[724-1,062] \mathrm{mL} / \mathrm{m}^{2}, p=0.722$; non-SIMD with SW $\leq 13 \%, 853[754-1,170] \mathrm{mL} / \mathrm{m}^{2} \mathrm{vs}$. non-SIMD with SW >13\%, 795 [730-1,007] mL/m², $p=0.289)$. SIMD, sepsis-induced myocardial dysfunction; GEDI, global end-diastolic volume index; SW, stroke volume variation. 
defined SIMD by LVEF measured by transthoracic echocardiography. The threshold value of LVEF for SIMD was defined as $50 \%$ based on the previous studies $[3,8,14]$.

In our results, there was no significant difference in GEDI between patients with SIMD and non-SIMD on the day of enrollment. Although the extent of fluid resuscitation was not known, these GEDI values were similar to the results reported by Eichhorn et al. [31]. Contrary to our expectation, the GEDI in patients with SIMD was not necessarily greater than that in patients without SIMD during early sepsis.

Analysis of the relationship between GEDI and SVV showed that the correlation was paradoxical in the SIMD group on the day of enrollment. In the second analysis, we found that on the day of enrollment, the GEDI in patients with SVV $\leq 13 \%$ was significantly lower than that in patients with SVV $>13 \%$ in the SIMD group. These findings are different from the generally recognized relationship between GEDI and SVV; this implies that greater GEDI is usually associated with lower SVV according to adequate cardiac filling. Although several interpretations for this paradoxical relationship may be considered-such as a limitation of the static parameters (GEDI) to predict fluid responsiveness compared with the dynamic parameters (SVV) $[19,20]$, a large interindividual variance of GEDI [32], the racial difference that the Japanese have smaller hearts than non-Asians [15], and the presence of LV diastolic dysfunction that impairs compensatory LV enlargement [4]-none of these explanations are definitive.

In contrast, there was no significant difference between GEDI in patients with SVV $\leq 13 \%$ and SVV $>13 \%$ among the non-SIMD group on the day of enrollment, and the same results were found between both SVV groups on day 2, regardless of the LVEF. These findings are also different from the generally recognized relationship between GEDI and SVV mentioned above. Since we were unable to identify a definite relationship between GEDI and SVV in patients with or without SIMD, the optimal GEDI value for these patients could not be defined in the present study.

\section{Limitations}

This subgroup analysis has several limitations. This is a retrospective analysis with a small sample size. Since the original study excluded patients with more than 5 days from the onset of acute respiratory failure, the exact date of occurrence of sepsis in each patient was unknown [13]. The ventilator settings, such as tidal volume (reliable when tidal volume is at least $8 \mathrm{mg} / \mathrm{kg}$ ), spontaneous mode (pressure support ventilation), and airway pressure release ventilation mode, may have influenced SVV measurement $[33,34]$. In this study, the ventilator mode depended on each institution's policy; therefore, SVV as a predictor of fluid responsiveness was not an adequate measurement for some patients. Although the use of muscle relaxants may affect the SVV, we did not examine their use in the original study and could not evaluate their influence on SVV. In the protocol of the present study, the method used to measure LVEF by transthoracic echocardiography was not uniform. There was a possibility that LVEF in some patients with SIMD may have improved on day 2 and such an improvement could have affected the results of the present study.

\section{Conclusions}

During the early phase of patients with severe sepsis on mechanical ventilation, there was no constant relationship between GEDI and fluid reserve responsiveness, irrespective of the presence of SIMD, defined as LVEF $\leq 50 \%$. Since the absolute value of optimal GEDI, indicating adequate cardiac filling, may not be determined regardless of the presence of SIMD, GEDI should be used as a cardiac preload parameter with awareness of its limitations.

\section{Abbreviations}

ALI: Acute lung injury; ARDS: Acute respiratory distress syndrome;

EVLW: Extravascular lung water; EVLWi: Extravascular lung water index; GEDI: Global end-diastolic volume index; GEDV: Global end-diastolic volume; LV: Left ventricular; LVEF: Left ventricular ejection fraction; PPV: Pulse pressure variation; PVPI: Pulmonary vascular permeability index; SIMD: Sepsis-induced myocardial dysfunction; SW: Stroke volume variation; TD: Thermodilution.

\section{Competing interests}

Dr. Taira is a member of the medical advisory board of Pulsion Medical Systems. The other authors declare that they have no competing interests.

\section{Authors' contributions}

All authors conceived and designed the study, wrote the study protocol, and acquired the clinical data. TE and SK were responsible for the statistical analyses and the first draft of the manuscript. All authors amended and commented on the manuscript and approved the final version.

\section{Acknowledgements}

This work was supported in part by a Grant-in-Aid for Scientific Research (22592023) from the Ministry of Education, Science, Sports, and Culture of Japan.

\section{Author details}

'Department of Emergency and Critical Care Medicine, Tohoku University Hospital, Sendai, Miyagi 980-8574, Japan. ²Division of Emergency Medicine, Tohoku University Graduate School of Medicine, Sendai, Miyagi 980-8574, Japan. ${ }^{3}$ Department of Emergency and Critical Care Medicine, Kurume University School of Medicine, Kurume-shi, Fukuoka 830-0011, Japan. ${ }^{4}$ Department of Emergency and Critical Care Medicine, Faculty of Medicine, Fukuoka University, Fukuoka, Fukuoka 814-0180, Japan. ${ }^{5}$ Department of Emergency and Critical Care Medicine, Kansai Medical University, Moriguchi, Osaka 570-8506, Japan. ${ }^{6}$ Department of Emergency and Critical Care Medicine, St. Marianna University School of Medicine, Kawasaki, Kanagawa 216-8511, Japan. 7 Department of Emergency and Critical Care Medicine, Nara Medical University, Kashihara, Nara 634-8521, Japan. ${ }^{8}$ Department of Emergency and Critical Care Medicine, Nippon Medical School Hospital, Bunkyo-ku, Tokyo 113-8603, Japan. ${ }^{9}$ Department of Emergency and Critical Care Medicine, Nihon University School of Medicine Itabashi Hospital, Itabashi-ku, Tokyo 173-8610, Japan. ${ }^{10}$ Shock Trauma and Emergency Medical Center, Tokyo Medical and Dental University Hospital of Medicine, Bunkyo-ku, Tokyo 113-8519, Japan. ${ }^{11}$ Department of Emergency and Critical Care Medicine, Juntendo University Nerima Hospital, Nerima-ku, Tokyo 177-8521, Japan. ${ }^{12}$ Department of Critical Care Medicine, Jikei University 
School of Medicine, Minato-ku, Tokyo 105-8471, Japan. ${ }^{13}$ Emergency and Critical Care Medicine, National Hospital Organization Disaster Medical Center, Tachikawa-shi, Tokyo 190-0014, Japan. ${ }^{14}$ Department of Intensive Care Medicine, Saiseikai Yokohamashi Tobu Hospital, Kanagawa, Japan. ${ }^{15}$ Department of Emergency and Critical Care Medicine, Social Insurance Chukyo Hospital, Nagoya, Aichi 457-8510, Japan. ${ }^{16}$ Advanced Emergency and Critical Care Center, Kansai Medical University Takii Hospital, Moriguchi, Osaka 570-8507, Japan. ${ }^{17}$ Emergency and Critical Care Medical Center, Osaka City General Hospital, Miyakojima, Osaka 534-0021, Japan. ${ }^{18}$ Intensive Care Unit, Kobe City Medical Center General Hospital, Kobe, Hyogo 650-0046, Japan. ${ }^{19}$ Department of Anesthesia and Intensive Care, Hiroshima City Hospital, Hiroshima-shi, Hiroshima 730-8518, Japan. ${ }^{20}$ Advanced Medical Emergency and Critical Care Center, Yamaguchi University Hospital, Ube, Yamaguchi 755-8505, Japan. ${ }^{21}$ Intensive Care Unit, Nagasaki University Hospital, Sakamoto, Nagasaki 852-8501, Japan. ${ }^{22}$ Department of Emergency and Critical Care Medicine, Nippon Medical School Tama Nagayama Hospital, Tama-shi, Tokyo 206-8512, Japan. ${ }^{23}$ Department of Emergency and Critical Care Medicine, Nippon Medical School Chiba Hokusou Hospital, Inzai-shi, Chiba 270-1694, Japan.

Received: 31 July 2013 Accepted: 7 November 2013

Published: 28 November 2013

\section{References}

1. Vieillard-Baron A: Septic cardiomyopathy. Ann Intensive Care 2011, 1:6.

2. Jardin F, Brun-Ney D, Auvert B, Beauchet A, Bourdarias JP: Sepsis-related cardiogenic shock. Crit Care Med 1990, 18:1055-1060.

3. Bouhemad B, Nicolas-Robin A, Arbelot C, Arthaud M, Feger F, Rouby JJ: Acute left ventricular dilatation and shock-induced myocardial dysfunction. Crit Care Med 2009, 37:441-447.

4. Romero-Bermejo FJ, Ruiz-Bailen M, Gil-Cebrian J, Huertos-Ranchal MJ: Sepsis-induced cardiomyopathy. Current Cardiol Rev 2011, 7:163-183.

5. Parker MM, Shelhamer JH, Bacharach SL, Green MV, Natanson C, Frederick TM, Damske BA, Parrillo JE: Profound but reversible myocardial depression in patients with septic shock. Ann Intern Med 1984, 100:483-490.

6. Jardin F, Fourme T, Page B, Loubieres Y, Vieillard-Baron A, Beauchet A, Bourdarias JP: Persistent preload defect in severe sepsis despite fluid loading: a longitudinal echocardiographic study in patients with septic shock. Chest 1999, 116:1354-1359.

7. Vieillard-Baron A, Caille V, Charron C, Belliard G, Page B, Jardin F: Actual incidence of global left ventricular hypokinesia in adult septic shock. Crit Care Med 2008, 36:1701-1706.

8. Charpentier J, Luyt CE, Fulla Y, Vinsonneau C, Cariou A, Grabar S, Dhainaut JF, Mira JP, Chiche JD: Brain natriuretic peptide: a marker of myocardial dysfunction and prognosis during severe sepsis. Crit Care Med 2004, 32:660-665.

9. Schneider AJ, Teule GJ, Groeneveld AB, Nauta J, Heidendal GA, Thijs LG: Biventricular performance during volume loading in patients with early septic shock, with emphasis on the right ventricle: a combined hemodynamic and radionuclide study. Am Heart J 1988, 116:103-112.

10. Furian T, Aguiar C, Prado K, Ribeiro RV, Becker L, Martinelli N, Clausell N, Rohde $L E$, Biolo A: Ventricular dysfunction and dilation in severe sepsis and septic shock: relation to endothelial function and mortality. J Crit Care 2012, 27(319):e9-e15.

11. Dellinger RP, Levy MM, Rhodes A, Annane D, Gerlach H, Opal SM, Sevransky JE, Sprung CL, Douglas IS, Jaeschke R, Osborn TM, Nunnally ME, Townsend SR, Reinhart K, Kleinpell RM, Angus DC, Deutschman CS, Machado FR, Rubenfeld GD, Webb SA, Beale RJ, Vincent IL, Moreno R, Surviving Sepsis Campaign Guidelines Committee including the Pediatric Subgroup: Surviving sepsis campaign: international guidelines for management of severe sepsis and septic shock: 2012. Crit Care Med 2013, 41:580-637.

12. Michard F, Alaya S, Zarka V, Bahloul M, Richard C, Teboul JL: Global enddiastolic volume as an indicator of cardiac preload in patients with septic shock. Chest 2003, 124:1900-1908.

13. Kushimoto S, Taira Y, Kitazawa Y, Okuchi K, Sakamoto T, Ishikura H, Endo T, Yamanouchi S, Tagami T, Yamaguchi J, Yoshikawa K, Sugita M, Kase Y, Kanemura T, Takahashi H, Kuroki Y, Izumino H, Rinka H, Seo R, Takatori M, Kaneko T, Nakamura T, Irahara T, Saito N, Watanabe A, The PiCCO Pulmonary Edema Study Group: The clinical usefulness of extravascular lung water and pulmonary vascular permeability index to diagnose and characterize pulmonary edema: a prospective multicenter study on the quantitative differential diagnostic definition for acute lung injury/acute respiratory distress syndrome. Crit Care 2012, 16:R232.

14. Landesberg G, Gilon D, Meroz Y, Georgieva M, Levin PD, Goodman S, Avidan A, Beeri R, Weissman C, Jaffe AS, Sprung CL: Diastolic dysfunction and mortality in severe sepsis and septic shock. Eur Heart J 2012, 33:895-903.

15. Daimon $M$, Watanabe $H$, Abe $Y$, Hirata $K$, Hozumi T, Ishii K, Ito H, Iwakura $K$, Izumi C, Matsuzaki M, Minagoe S, Abe H, Murata K, Nakatani S, Negishi K, Yoshida K, Tanabe K, Tanaka N, Tokai K, Yoshikawa J, JAMP Study Investigators: Normal values of echocardiographic parameters in relation to age in a healthy Japanese population: the JAMP study. Circ J 2008, 72:1859-1866.

16. Michard F, Boussat S, Chemla D, Anguel N, Mercat A, Lecarpentier Y, Richard C, Pinsky MR, Teboul JL: Relation between respiratory changes in arterial pulse pressure and fluid responsiveness in septic patients with acute circulatory failure. Am J Respir Crit Care Med 2000, 162:134-138.

17. Monnet X, Teboul JL: Volume responsiveness. Current Opin Critic Care 2007, 13:549-553.

18. Hofer CK, Muller SM, Furrer L, Klaghofer R, Genoni M, Zollinger A: Stroke volume and pulse pressure variation for prediction of fluid responsiveness in patients undergoing off-pump coronary artery bypass grafting. Chest 2005, 128:848-854.

19. Broch O, Renner J, Gruenewald M, Meybohm P, Hocker J, Schottler J, Steinfath M, Bein B: Variation of left ventricular outflow tract velocity and global end-diastolic volume index reliably predict fluid responsiveness in cardiac surgery patients. J Crit Care 2012, 27(325):e7-e13.

20. Marik PE, Cavallazzi R, Vasu T, Hirani A: Dynamic changes in arterial waveform derived variables and fluid responsiveness in mechanically ventilated patients: a systematic review of the literature. Crit Care Med 2009, 37:2642-2647.

21. Reuter DA, Felbinger TW, Schmidt C, Kilger E, Goedje O, Lamm P, Goetz AE: Stroke volume variations for assessment of cardiac responsiveness to volume loading in mechanically ventilated patients after cardiac surgery. Intensive Care Med 2002, 28:392-398.

22. Berkenstadt $H$, Margalit N, Hadani M, Friedman Z, Segal E, Villa Y, Perel A Stroke volume variation as a predictor of fluid responsiveness in patients undergoing brain surgery. Anesth Analg 2001, 92:984-989.

23. Zhang Z, Lu B, Sheng $X$, Jin N: Accuracy of stroke volume variation in predicting fluid responsiveness: a systematic review and meta-analysis. J Anesthesia 2011, 25:904-916.

24. Hofer CK, Cannesson M: Monitoring fluid responsiveness. Acta Anaesthesiol Taiwan 2011, 49:59-65.

25. Sakka SG, Ruhl CC, Pfeiffer UJ, Beale R, McLuckie A, Reinhart K, MeierHellmann A: Assessment of cardiac preload and extravascular lung water by single transpulmonary thermodilution. Intensive Care Med 2000, 26:180-187.

26. Monnet X, Anguel N, Osman D, Hamzaoui O, Richard C, Teboul JL: Assessing pulmonary permeability by transpulmonary thermodilution allows differentiation of hydrostatic pulmonary edema from ALI/ARDS. Intensive Care Med 2007, 33:448-453.

27. Berkowitz DM, Danai PA, Eaton S, Moss M, Martin GS: Accurate characterization of extravascular lung water in acute respiratory distress syndrome. Crit Care Med 2008, 36:1803-1809.

28. Phillips CR, Chesnutt MS, Smith SM: Extravascular lung water in sepsisassociated acute respiratory distress syndrome: indexing with predicted body weight improves correlation with severity of illness and survival. Crit Care Med 2008, 36:69-73.

29. Craig TR, Duffy MJ, Shyamsundar M, McDowell C, McLaughlin B, Elborn JS, McAuley DF: Extravascular lung water indexed to predicted body weight is a novel predictor of intensive care unit mortality in patients with acute lung injury. Crit Care Med 2010, 38:114-120.

30. Boldt J: Clinical review: hemodynamic monitoring in the intensive care unit. Crit Care 2002, 6:52-59.

31. Eichhorn V, Goepfert MS, Eulenburg C, Malbrain ML, Reuter DA: Comparison of values in critically ill patients for global end-diastolic volume and extravascular lung water measured by transcardiopulmonary thermodilution: a meta-analysis of the literature. Med Intensiva 2012, 36:467-474.

32. Wolf S, Riess A, Landscheidt JF, Lumenta CB, Friederich P, Schurer L: Global end-diastolic volume acquired by transpulmonary thermodilution depends on age and gender in awake and spontaneously breathing patients. Crit Care 2009, 13:R202. 
33. Reuter DA, Bayerlein J, Goepfert MS, Weis FC, Kilger E, Lamm P, Goetz AE: Influence of tidal volume on left ventricular stroke volume variation measured by pulse contour analysis in mechanically ventilated patients. Intensive Care Med 2003, 29:476-480.

34. Perner A, Faber T: Stroke volume variation does not predict fluid responsiveness in patients with septic shock on pressure support ventilation. Acta Anaesthesiol Scand 2006, 50:1068-1073.

doi:10.1186/2052-0492-1-11

Cite this article as: Endo et al.: Limitations of global end-diastolic

volume index as a parameter of cardiac preload in the early phase of severe sepsis: a subgroup analysis of a multicenter, prospective observational study. Journal of Intensive Care 2013 1:11.

\section{Submit your next manuscript to BioMed Central and take full advantage of:}

- Convenient online submission

- Thorough peer review

- No space constraints or color figure charges

- Immediate publication on acceptance

- Inclusion in PubMed, CAS, Scopus and Google Scholar

- Research which is freely available for redistribution 